\title{
Structure of exotic nuclei from direct reactions
}

A. Gillibert ${ }^{\mathrm{a}}$, N. Alamanos ${ }^{\mathrm{a}}$, M. Alvarez ${ }^{\mathrm{a}}$, F. Auger ${ }^{\mathrm{a}}$, D. Beaumel ${ }^{\mathrm{b}}$, E. Becheva $^{\mathrm{b}}, \mathrm{Y}$.

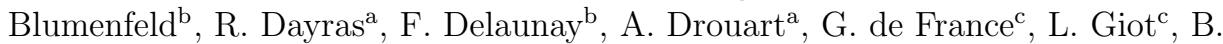
Jurado $^{c}$, N. Keeley ${ }^{\mathrm{a} *}$, K.W. Kemperd ${ }^{\mathrm{d}}$, V. Lapoux ${ }^{\mathrm{a}}$, W. Mittig ${ }^{\mathrm{c}}$, X. Mougeot, L. Nalpas ${ }^{\mathrm{a}}$, A. Obertelli ${ }^{\mathrm{a}}$, N. Patronis ${ }^{\mathrm{e}}$, A. Pakou ${ }^{\mathrm{e}}$, E.C. Pollacco ${ }^{\mathrm{a}}$, R. Raabe ${ }^{\mathrm{a}}$, P. Roussel-Chomaz ${ }^{\mathrm{c}}$, F. Rejmund ${ }^{\text {, }}$, M. Rejmund ${ }^{\mathrm{c}}$, H. Savajols ${ }^{\mathrm{c}}$, J.A. Scarpaci ${ }^{\mathrm{b}}$, J.L. Sida ${ }^{\mathrm{a}}$, F. Skaza ${ }^{\mathrm{a}}$, S. Stepantsov $^{f}$, Ch. Theisen ${ }^{\mathrm{a}}$ and R. Wolski ${ }^{\mathrm{g}}$

${ }^{a}$ DAPNIA/SPhN, CE Saclay, 91191 Gif-sur-Yvette, France

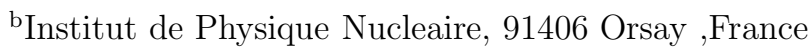

${ }^{\mathrm{c}}$ GANIL, BP 5027, 14021 Caen Cedex, France

${ }^{\mathrm{d}}$ Department of Physics, Florida State University, Florida 32306-4350 USA

${ }^{\mathrm{e} U n i v e r s i t y ~ o f ~ I o a n n i n a, ~} 45110$ Ioannina, Greece

${ }^{\mathrm{f}}$ JINR,FLNR 141980 Dubna, Russia

'The Henryk Niewodniczanski Institute of Nuclear Physics, Krakow, Poland

\section{Introduction}

Direct reactions with stable beams have been for years the origin of major developments in nuclear structure with the achievement of the shell model for stable nuclei. With the new radioactive beams, a new degree of freedom, the isospin, is now currently investigated to improve that knowledge and find new phenomena and properties of the nuclear matter, like neutron halo or neutron skin, the evolution of spin-orbit or the evolution of shell closures far from stability. In the following, we will illustrate the physics adressed by direct reactions and the experimental tools to investigate inverse kinematics in two cases: the structure of ${ }^{8} \mathrm{He}$ and the study of $\mathrm{N}=16$ shell closure for neutron rich nuclei.

\section{Study of the ${ }^{8} \mathrm{He}$ wave function}

Large spatial extensions of the neutron distributions have been found for ${ }^{6} \mathrm{He}$ and ${ }^{8} \mathrm{He}$, producing a halo in the case of ${ }^{6} \mathrm{He}[1]$ and a possible neutron skin for ${ }^{8} \mathrm{He}[2]$. From reaction cross sections [3] and high energy proton elastic scattering [4], it is shown that ${ }^{8} \mathrm{He}$ with a matter rms radius $2.5 \mathrm{fm}$ has almost the same size as ${ }^{6} \mathrm{He}$, although having two

${ }^{*}$ N. K. gratefully acknowledges the receipt of a Marie Curie Intra-European Fellowship from the European Commisssion. 
neutrons more. ${ }^{8} \mathrm{He}$ is generally described as the 5 body COSMA[2] model does : an inert $\alpha$ core surrounded by four valence neutrons. The neutron $1 \mathrm{p} 3 / 2$ subshell is fully occupied, which results in a compact nucleus and the neutron-skin structure. In the $2 \mathrm{n}$ subsystem, this corresponds to a description dominated by ${ }^{6} \mathrm{He}\left(2^{+}\right)+2 \mathrm{n}$, whereas ${ }^{6} \mathrm{He}\left(0^{+}\right)+2 \mathrm{n}$ is negligible. The $2 \mathrm{n}$ transfer ${ }^{8} \mathrm{He}(\mathrm{p}, \mathrm{t}){ }^{6} \mathrm{He}$ is expected to provide information on that point.

Elastic and inelastic proton scattering is a good tool to test the matter distribution, the transition densities and the spectroscopy of weakly bound nuclei. This was done with the ${ }^{8} \mathrm{He}$ beam now available at GANIL. A new experimental set-up has been developed, so that all the channels (elastic, inelastic and transfer) were simultaneously measured in the same conditions.

\subsection{Experimental set-up}

The ${ }^{8} \mathrm{He}+\mathrm{p}$ experiment[5] was done at GANIL with a ${ }^{8} \mathrm{He}$ beam delivered by the SPIRAL facility at $15.7 \mathrm{~A} . \mathrm{MeV}$ and a $\mathrm{CH}_{2}$ target. The heavy ejectiles were measured in a wall of plastic scintillator at forward angles. The recoiling nuclei $\left({ }^{1} \mathrm{H}\right.$ for elastic/inelastic scattering and ${ }^{2} \mathrm{H},{ }^{3} \mathrm{H}$ for transfer) were detected in coincidence in the MUST device [6], an array of $8 \mathrm{Si}$-strip and $\mathrm{Si}(\mathrm{Li})$ telescopes $\left(6 \times 6 \mathrm{~cm}^{2}\right.$ each) at $15 \mathrm{~cm}$ from the target. Two beam tracking detectors [7] were necessary to define the impact of the beam on the target and improve the angular resolution. The scattering angle in the laboratory frame $\theta_{l a b}$ and the energy $\mathrm{E}$ of the recoiling nuclei, reconstructed event by event, are plotted in fig. 2 of [5]. The importance of the $1 \mathrm{n}$ transfer channel is obvious.

\subsection{Elastic scattering}

The angular distribution for elastic scattering is shown in fig.1. As a first step, we tried to analyze the data without coupling to the $1 \mathrm{n}$ transfer channel. The potential was calculated with the microscopic complex JLM [8] nucleon-nucleus potential, using the ${ }^{8} \mathrm{He}$ gs densities generated by the NCSM calculations [9]. Data are poorly reproduced with the usual parameter set $\lambda_{v}=1.0$ and $\lambda_{w}=0.8$ (dashed line). A better agreement may be obtained if $\lambda_{v}$ and $\lambda_{w}$ are left as free parameters (full line). However, even with uncommon values, the elastic scattering at forward angles is still not well reproduced . This is an indication that the renormalization of volume potentials by $\lambda_{v}$ and $\lambda_{w}$ is not an appropriate method in that case when a dynamical polarization potential is needed.

\subsection{Coupling to the $1 \mathrm{n}$ transfer reaction}

Considering the large cross sections for the 1 transfer reaction compared to elastic scattering (fig.2 left), it is more exact to use the coupled reaction channels (CRC) formalism with the coupling scheme shown in fig. 2 (right). It is necessary to take into account the deuteron break-up in the exit channel which is done in the CDCC formalism. The unknown $\mathrm{d}+{ }^{7} \mathrm{He}$ potential is obtained by folding the nucleon-nucleus global potential from $[10]$.

It is worth noting the very nice agreement obtained for elastic scattering over the whole angular range, both at small angles and for the first minimum at 65 degrees. With the gs densities given by the NCSM model, the rms radii for the proton, neutron and matter distributions are respectively $2.00,2.59$ and $2.46 \mathrm{fm}$. The resulting neutron-skin thickness is $\left\langle r^{2}>_{n}^{1 / 2}-<r^{2}>_{p}^{1 / 2}=0.6 \mathrm{fm}\right.$ compared to $1 \mathrm{fm}$ for the COSMA model[2]. 


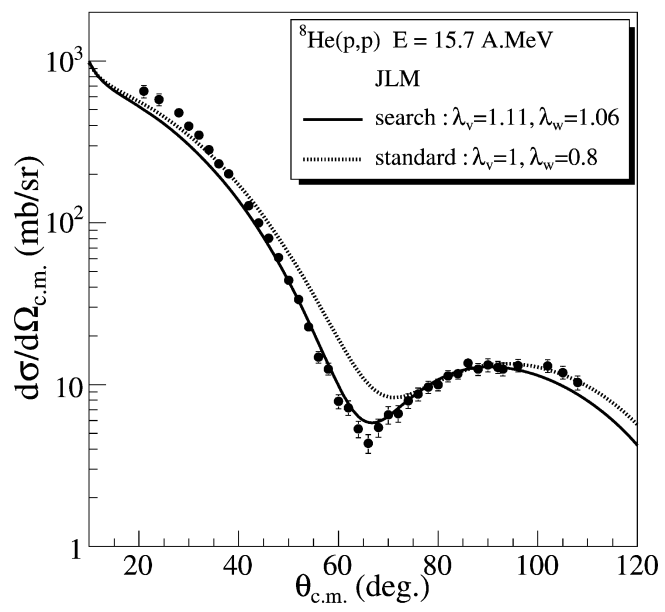

Figure 1. Angular distributions for proton elastic scattering with ${ }^{8} \mathrm{He}$ projectile at 15.7 A.MeV. Calculations with the JLM complex microscopical potential were done with the two parameters $\lambda_{v}$ and $\lambda_{w}$. The dashed line corresponds to the standard parameter set for light nuclei. $\lambda_{v}$ and $\lambda_{w}$ were also varied to obtain the best agreement corresponding to the full line.
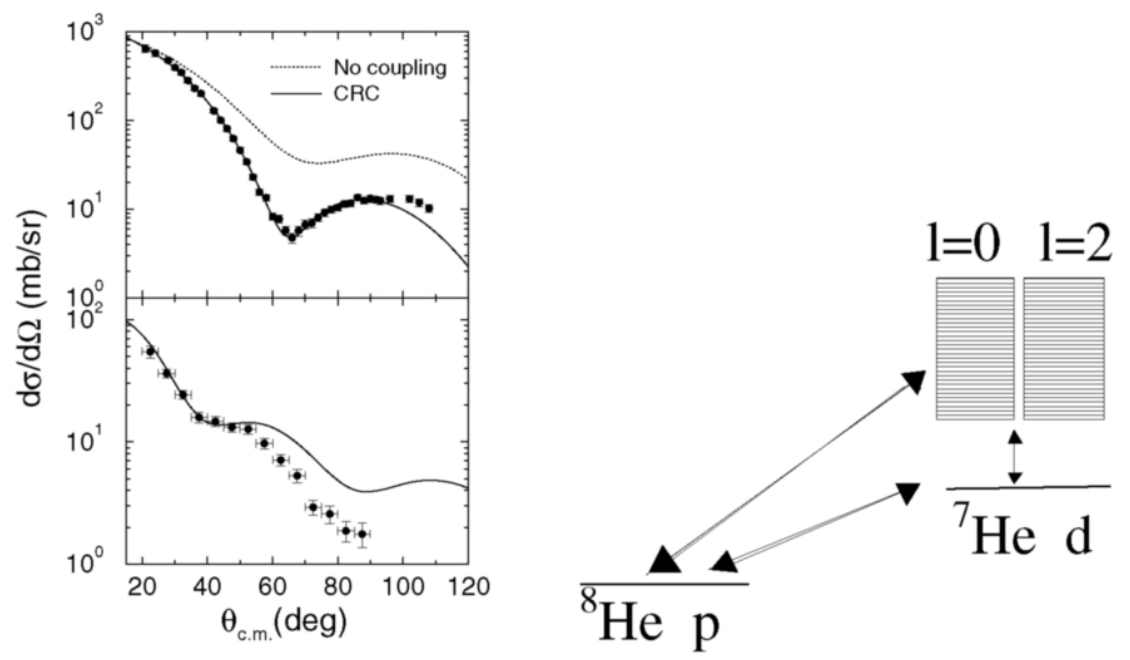

Figure 2. (left) Angular distributions for elastic scattering ${ }^{8} \mathrm{He}$ (p,p) ${ }^{8} \mathrm{He}$ (upper) and the $1 \mathrm{n}$ transfer reaction (lower) ${ }^{8} \mathrm{He}(\mathrm{p}, \mathrm{d}){ }^{7} \mathrm{He}$; the coupling scheme used in the CRC calculation is shown on the right. 

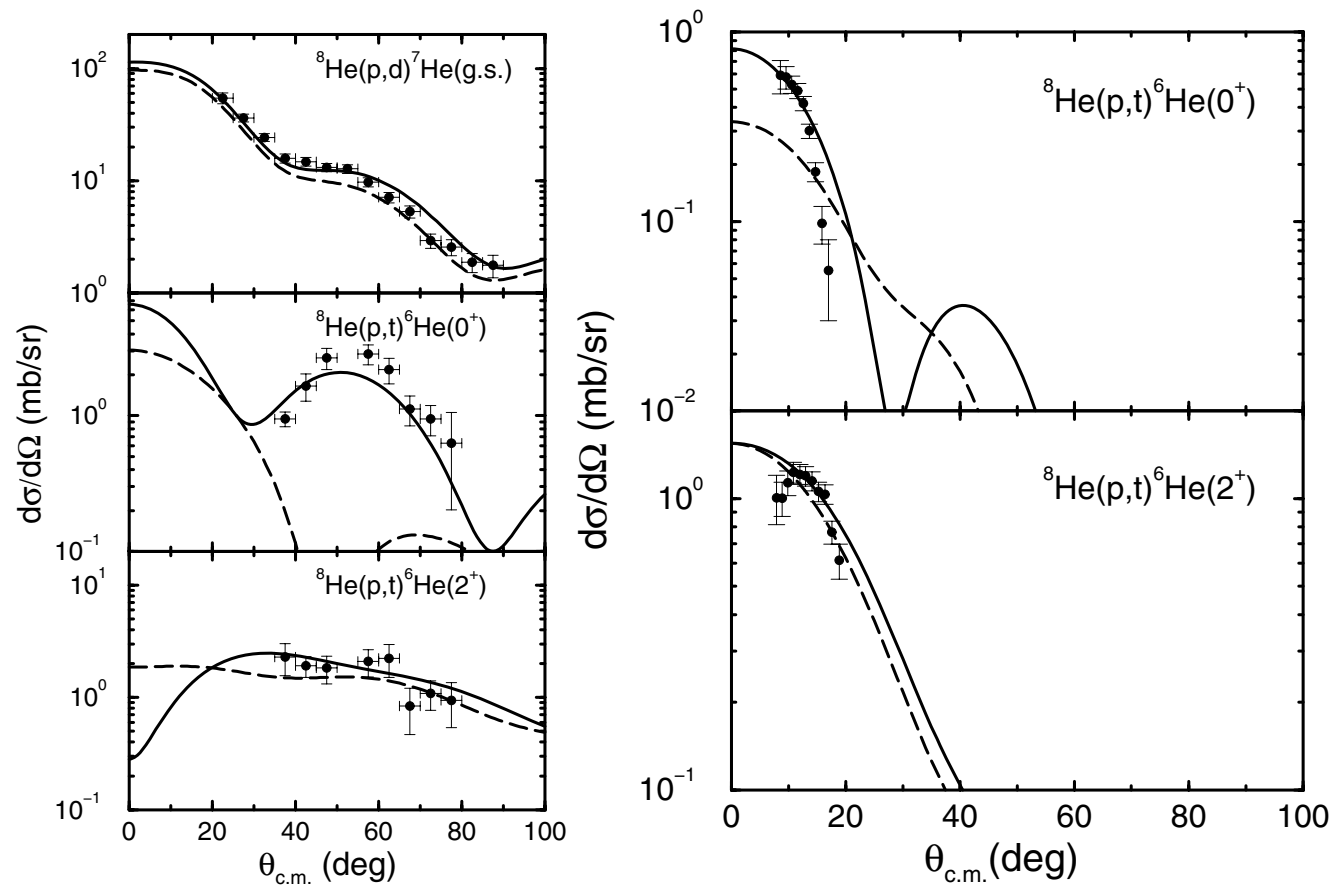

Figure 3. Angular distributions obtained for data set at two different incident energies (left) 15.7 A.MeV and (right) 63 A.MeV.

The 1n transfer channel angular distribution is also well reproduced, considering that no effort was made to reproduce angles larger than 60 degrees by a tuning of the Koning Delaroche potential, since the spectroscopic factors are mainly sensitive to the small angle domain. In that calculation, the spectroscopic factor $C^{2} S$ was found to be 3.3, not far but significantly different from 4 , the expected value from a $\nu(p 3 / 2)^{4}$ configuration, as predicted by the COSMA model.

\subsection{Analysis of the $2 \mathrm{n}$ transfer reaction}

The $2 \mathrm{n}$ transfer reaction ${ }^{8} \mathrm{He}(\mathrm{p}, \mathrm{t}){ }^{6} \mathrm{He}$ has been measured at $61.3 \mathrm{~A} . \mathrm{MeV}$ at Riken[11]. Angular distributions were obtained for the transfer to the ground state ${ }^{6} \mathrm{He}\left(0^{+}\right)$and first excited state ${ }^{6} \mathrm{He}\left(2^{+}\right)$. In the DWBA analysis, the cross sections were reproduced with a spectroscopic factor ratio $\mathrm{R}=<^{8} \mathrm{He}\left|{ }^{6} \mathrm{He}\left(\mathrm{0}^{+}\right)>/<^{8} \mathrm{He}\right|{ }^{6} \mathrm{He}\left(2^{+}\right)>$approximately equal to one. This corresponds in the $2 \mathrm{n}$ subsystem to a wave function for ${ }^{8} \mathrm{He}$ dominated by a configuration ${ }^{6} \mathrm{He}\left(2^{+}\right)+2 n$, the four valence neutrons in the p3/2 subshell $(p 3 / 2)^{4}$ and a closed subshell compact ${ }^{8} \mathrm{He}$, as predicted by the COSMA model. The other projection ${ }^{6} \mathrm{He}\left(0^{+}\right)+2 n$ corresponding to the $\nu(p 3 / 2)^{2}(p 1 / 2)^{2}$ configuration would be negligible.

In our experiment, all the reactions (elastic, inelastic, $1 \mathrm{n}$ and $2 \mathrm{n}$ transfer) were measured at the same time with the same experimental set-up at the same beam energy, which 
removes many biases. The angular distributions for $1 \mathrm{n}$ and $2 \mathrm{n}$ transfer are shown in fig.3 (left). As already stated, the coupling to the $1 \mathrm{n}$ transfer is quite strong, and we have analyzed our data in the CRC formalism with the couplings shown in fig.2(right), plus direct and two-step $2 \mathrm{n}$ transfer[12] . Moreover we have included the data set at 61.3 A.MeV to take into account all the existing data and constraints on the calculation. Many parameters are involved in the calculation, some are measured, some may be fixed from external considerations, some are unknown and have been approximated. The goal is to describe all the data with only two free parameters, the spectroscopic amplitude overlaps $<\left.^{8} \mathrm{He}\right|^{6} \mathrm{He}\left(0^{+}\right)>$and $<\left.^{8} \mathrm{He}\right|^{6} \mathrm{He}\left(2^{+}\right)>$equivalent to the ratio $\mathrm{R}$ of the spectroscopic factors. If the reaction mechanism is overall under control, reliable structure information will be obtained. In fig. 3 are shown the results of the calculations for the transfer channels at both energies. The most sensitive data turn out to be the $2 \mathrm{n}$ transfer to the gs ${ }^{6} \mathrm{He}\left(0^{+}\right)$ distributions at both energies. With the ratio $\mathrm{R}=1$, as proposed in [11], that transfer is not reproduced. An overall agreement is obtained for a much higher value, $\mathrm{R}=20$. The main uncertainty remains the potential ${ }^{6} \mathrm{He}+\mathrm{t}$ approximated by data on ${ }^{6} \mathrm{Li}+$ ${ }^{3} \mathrm{He}$. However, in all the checks, the ratio $\mathrm{R}$ remains significantly higher than 1 . That means even if the valence neutrons in ${ }^{8} \mathrm{He}$ mainly occupy the p3/2 subshell, we probe with the $2 \mathrm{n}$ transfer a part of the wave function where the $\mathrm{p} 1 / 2$ subshell may be also partly occupied. It would be quite interesting to fix as input to CRC calculations the spectroscopic amplitudes obtained from an ab-initio calculation like [13].

\section{One neutron transfer reaction and $\mathrm{N}=16$}

The evolution of shell effects with neutron excess is a topic of large interest. $\mathrm{N}=16$ has been proposed recently to be a new magic number far from stability. First indications came from the unbound ${ }^{26} \mathrm{O}$ and ${ }^{28} \mathrm{O}$ nuclei[14], mass surfaces with discontinuities observed at $\mathrm{N}=16$ for large neutron excesses[15] or the structure of ${ }^{23} \mathrm{O}$ ground state described as a single particle state $\mathrm{s}_{1 / 2}[16]$. Moreover, the first $2^{+}$excited state of ${ }^{24} \mathrm{O}$ was suggested to be at high excitation energy, larger than 3.6 MeV[17]. New calculations[18] suggest an enhancement with isospin of the gap between the $\mathrm{s}_{1 / 2}$ and $\mathrm{d}_{3 / 2}$ subshells of the sd neutron shell. The attractive interaction between protons in the $d_{5 / 2}$ and neutrons in the $d_{3 / 2}$ subshells seems to be a key point. Then, removing protons from ${ }^{30} \mathrm{Si}$ to ${ }^{24} \mathrm{O}$ makes the neutron $\mathrm{d}_{3 / 2}$ subshell less bound. Consequently, the last bound oxygen isotope ${ }^{24} \mathrm{O}$ could be a doubly magic nucleus, when ${ }^{28} \mathrm{O}$ is unbound in spite of $\mathrm{Z}=8$ and $\mathrm{N}=20$, the two magic numbers for stable nuclei.

More information may be obtained from the $\mathrm{N}=17$ isotones, since the study of the single particle excitations is well suited to determine the spacing between the $\mathrm{d}_{3 / 2}$ subshell and the fp shell. The lowest excitation energies of the negative parity states for the most bound $\mathrm{N}=17$ isotones $\left({ }^{35} \mathrm{Ar},{ }^{33} \mathrm{~S}\right.$ and $\left.{ }^{31} \mathrm{Si}\right)$ are quite high, around $3.5 \mathrm{MeV}$, reflecting the $\mathrm{N}=20$ major shell gap for stable nuclei. They are suddenly lower than $1.5 \mathrm{MeV}$ for ${ }^{29} \mathrm{Mg}[19]$. The next $\mathrm{N}=17$ isotone is ${ }^{27} \mathrm{Ne}$ for which little information[20] are available. As new Ne beams are now delivered by the SPIRAL facility at GANIL, we have studied ${ }^{27} \mathrm{Ne}$ with the one neutron transfer reaction ${ }^{26} \mathrm{Ne}(\mathrm{d}, \mathrm{p}){ }^{27} \mathrm{Ne}$ in inverse kinematics. 


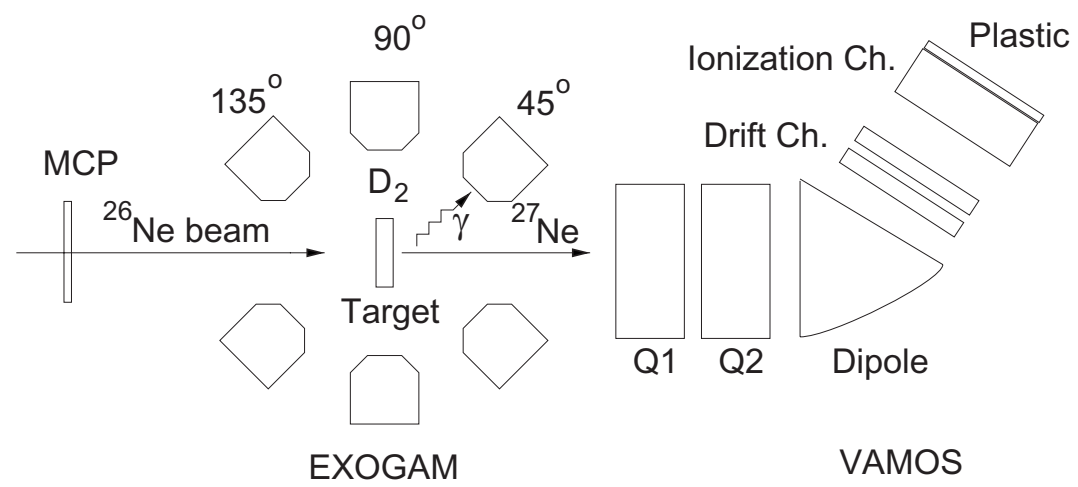

Figure 4. Experimental setup used for the one neutron transfer ${ }^{26} \mathrm{Ne}(\mathrm{d}, \mathrm{p}){ }^{27} \mathrm{Ne}$.

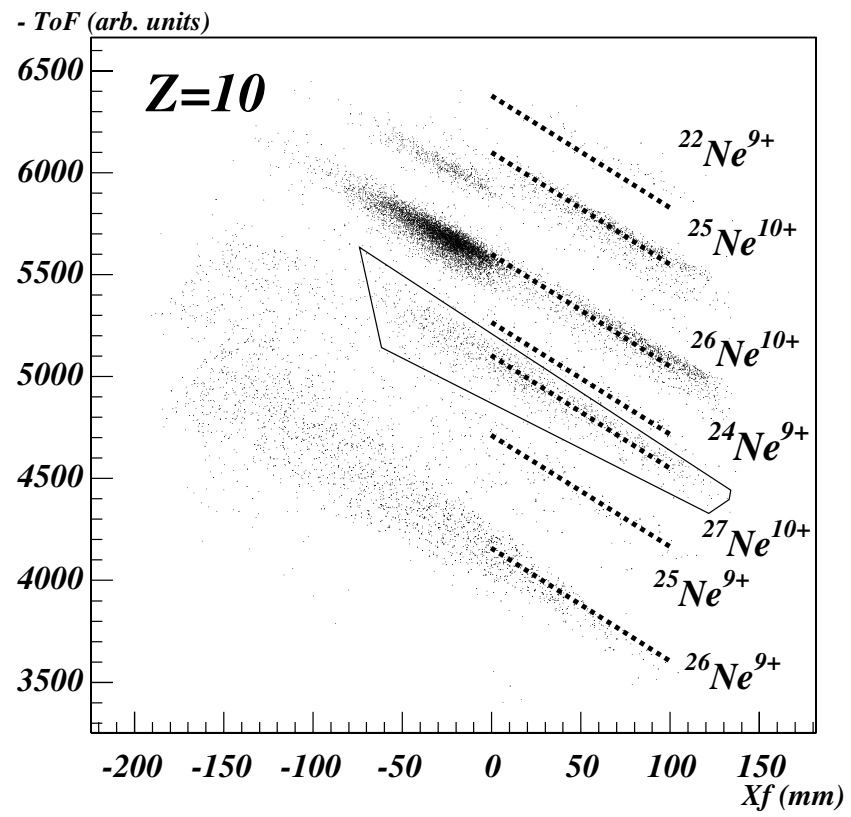

Figure 5. Identification of Ne isotopes transmitted in the focal plane of the VAMOS spectrometer with the transverse position in the focal plane versus a time of flight. The device is optimised for the one neutron transfer ${ }^{26} \mathrm{Ne}(\mathrm{d}, \mathrm{p}){ }^{27} \mathrm{Ne}$. 

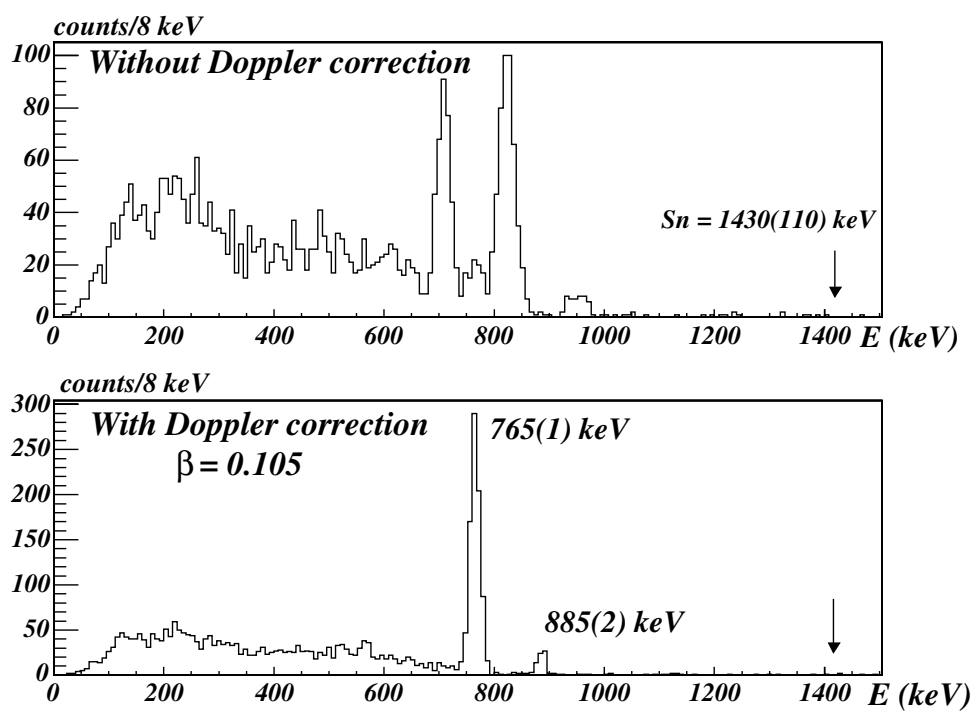

Figure 6. $\gamma$ spectrum corresponding to the one neutron transfer ${ }^{26} \mathrm{Ne}(\mathrm{d}, \mathrm{p}){ }^{27} \mathrm{Ne}$ before (up) and after (down) Doppler correction with $\beta=0.105$. Two transitions are observed at 765 and $885 \mathrm{keV}$ below the one neutron emission threshold $S_{n}$.

\subsection{Experimental set-up}

We used the Spiral beam ${ }^{26} \mathrm{Ne}$ at $9.7 \mathrm{MeV} /$ nucleon impinging on a $1 \mathrm{~mm}$ thick solid cryogenic deuterium target developed at GANIL[21]. The ejectiles were detected and identified with the VAMOS magnetic spectrometer [22]. Due to the large momentum acceptance and the inverse kinematics, not only ${ }^{27} \mathrm{Ne}^{10+}$ was transmitted, but other ejectiles simultaneously observed in the focal plane, originating from reaction channels like (d,d'), $(\mathrm{d}, \mathrm{n})$ or $(\mathrm{d}, \mathrm{t})[23]$. A complete identification was obtained with usual combinations of time of flight, energy loss and transverse position in the focal plane(fig.5). In the $1 \mathrm{n}$ transfer reaction $(\mathrm{d}, \mathrm{p})$, the recoiling protons are emitted at backward angles in the lab frame and a small kinetic energy for small $\mathrm{cm}$ angles. The target is very thick for such protons : not only the energy and angular stragglings are large, but the energy loss in the target is unknown since the reaction vertex is not reconstructed. For these reasons, we did not try to measure such recoiling protons.

In coincidence with the ejectiles, the emitted photons were measured with part of the EXOGAM spectrometer in a compact geometry around the target (fig.4). We used 11 clovers, placed at distance varying from 11 to $17 \mathrm{~cm}$ from the center of the target and three different values of the polar angle $\theta=45,90$ and 135 degrees relative to the beam axis in the laboratory frame. The absorption due to the whole target device affects mainly the efficiency of the ring at 90 degrees. The intrinsic resolution of the whole device was measured to be $2.6 \mathrm{keV}$ (FWHM) at $1332 \mathrm{keV}$, and the photopeak efficiency 

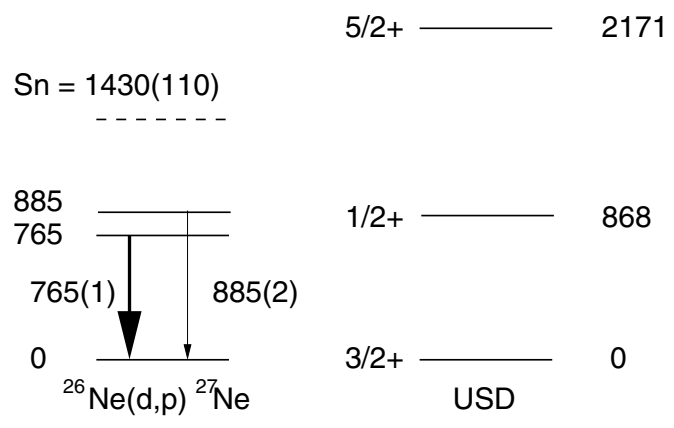

Figure 7. Spectroscopy of ${ }^{27} \mathrm{Ne}$ bound states (left part) observed in the one neutron transfer compared to the USD shell model predictions (right part).

was estimated to be $4.8 \%$. As the reaction vertex is unknown, only a mean value of the relative velocity $\beta=\mathrm{v} / \mathrm{c}$ may be used. The whole set-up and analysis have been tested with the inelastic scattering ${ }^{26} \mathrm{Ne}\left(\mathrm{d}, \mathrm{d}^{\prime}\right){ }^{26} \mathrm{Ne}$ to the well known first $2^{+}$excited state of ${ }^{26} \mathrm{Ne}$ and the $\gamma$-transition $2^{+} \rightarrow 0^{+}$at $2.02 \mathrm{MeV}$ which was fairly well reproduced for $\beta$ $=0.115$. That value was chosen so that the measured energies are equally well corrected for the Ge detectors at backward and forward angles. For ${ }^{27} \mathrm{Ne}$ and the (d,p) channel, a mean value $\beta=0.105$ was adopted.

\section{Results}

\section{1. $\gamma$ transitions and levels}

Below the one neutron emission threshold $\mathrm{S}_{1 n}=1430$ (110) keV, two transitions are observed at 765 and $885 \mathrm{keV}$ on the Doppler-corrected spectrum (fig.6). Within the limited statistics, we did not observe any coincidence between these transitions. Due to the low $\mathrm{S}_{1 n}$ value, it is the most likely that these transitions directly connect two excited states to the ground state as shown in fig.7. We did not observe a strong evidence for a transition at $120 \mathrm{keV}$ over the background, that would connect the $885 \mathrm{keV}$ to the 765 $\mathrm{keV}$ state. An upper limit of $20 \%$ was deduced for the branching ratio $\tau$, compared to the direct decay to the ground state. The deduced level scheme is summarized on fig.7 (left part) and compared to the predictions of the USD shell model [24] for the positive parity states of ${ }^{27} \mathrm{Ne}$. Only one positive parity state is predicted below $\mathrm{S}_{1 n}$, with spin $1 / 2^{+}$. The two experimental levels are consistent with that state, so that one of them may be a negative parity state.

\subsection{Cross sections and spectroscopic factors}

To go further, we performed calculations with the code FRESCO using the CRC (Coupled Reaction Channels), CDCC (Continuum Discretized Coupled Channels) and CC (Coupled Channels) formalisms [25], taking into account the deuteron break-up. The spectroscopic factors were obtained from the comparaison of calculated and experimental 
cross sections integrated in the VAMOS angular range for each $\gamma$ transition. The ground state cross section was deduced by difference between the inclusive cross section and the normalised cross sections in coincidence with $\gamma$ 's, with a larger relative uncertainty. The level at $765 \mathrm{keV}$ has a large cross section with a sizeable spectroscopic factor, whatever the angular momentum $\Delta \ell$ may be, 1 or 3 , in the considered angular range. The results are in good agreement with the experimental values(see table1 in [26]).

\subsection{Other results}

An other approach to produce and study ${ }^{27} \mathrm{Ne}$ is the one neutron break-up (equivalent to the $(\mathrm{d}, \mathrm{t})$ transfer channel) from a ${ }^{28} \mathrm{Ne}$ beam at higher incident energy. In that case, the results are sensitive to the wave function of ${ }^{28} \mathrm{Ne}$ with a competition between the normal and intruder states. Two experiments were done recently [27], [28] with the observation of two $\gamma$ transitions at energies fully consistent with our results. Due to the reaction mechanism, the two states are not populated like in our experiment, the peak at $885 \mathrm{keV}$ being even more intense than the $765 \mathrm{keV}$ one. For that reason, the transition at $120 \mathrm{keV}$ connecting the two states may be observed [28] over the background, with a measurement of the branching ratio $\tau=19.3 \%$, in agreement with our upper limit. In spite of a limited statistics, the momentum distribution of ${ }^{27} \mathrm{Ne}$ ejectiles was also measured[28], resulting in the determination of a transferred angular momentum $\Delta \ell$ lower than 2 , which excludes the possibility of a $f_{7 / 2}$ or $f_{5 / 2}$ state.

Moreover, assuming a $3 / 2^{+}$spin for the ground state as proposed by the shell model calculations, all these measurements done with $\gamma$-ray transitions are hardly able to measure a direct decay from a low-lying $7 / 2^{-}$state to the ground state. Such a low energy M2 transition has a long lifetime, which results in a large forward-backward asymetry not seen in the data.

Finally, combining all data and constraints, the $765 \mathrm{keV}$ state is consistent with a low energy negative parity state, with spin $\left(1 / 2^{-}, 3 / 2^{-}\right)$. Compared to the result of the SDFP-M calculation [28], [29] it may be a $3 / 2^{-}$state whereas the $7 / 2^{-}$at low energy remains unobserved.

\subsection{Conclusions}

All the results are consistent with a negative parity state at low excitation energy in ${ }^{27} \mathrm{Ne}$, which is an indication of a reduced sd-fp gap. This may be due to a reduction of the sd-fp spherical shell gap or a deformation effect, or due to both effects. The neutronproton tensor interaction proposed by T.Otsuka [30] may be responsible for such an effect with the attractive $\pi d_{5 / 2^{+}}-\nu d_{3 / 2^{+}}$and repulsive $\pi d_{5 / 2^{+}}-\nu f_{7 / 2^{-}}$terms. The maximum effect is expected for ${ }^{24} \mathrm{O}$ for which the proton $\mathrm{d}_{5 / 2}$ subshell is empty. Therefore, a similar experiment done with a ${ }^{24} \mathrm{O}$ beam, ${ }^{24} \mathrm{O}(\mathrm{d}, \mathrm{p}){ }^{25} \mathrm{O}$, although experimentally difficult since ${ }^{25} \mathrm{O}$ is unbound, is highly desirable.

\section{REFERENCES}

1. A. Lagoyannis et al., Phys. Lett. B 518 (2001) 27.

2. M.V. Zhukov et al., Phys. Rev. C 50 (1994) R1.

3. J.A. Tostevin et al., Nucl. Phys. A 616 (1997) 418c.

4. J.S. Al-Khalili et al., Phys. Rev. C 57 (1998) 1846. 
5. F. Skaza et al., Phys. Rev. C 73 (2006) 044301.

6. Y. Blumenfeld et al., Nucl. Instr. and Meth. A 421 (1999) 471.

7. S. Ottini et al., Nucl. Instr. and Meth. A 431 (1999) 476.

8. J.P. Jeukenne et al., Phys. Rev. C 16 (1977) 80.

9. P. Navratil et al., Phys. Rev. C 57 (1998) 3119.

10. A.J. Koning, J.P. Delaroche, Nucl. Phys. A 713 (2003) 231.

11. A.A. Korsheninnikov et al., Phys. Rev. Lett. 90 (2003) 082501.

12. N. Keeley et al., submitted to Phys. Lett. B.

13. S.C. Pieper et al., Phys. Rev. C 70 (2004) 054325.

14. H. Sakurai et al., Phys. Lett. B 448 (1999) 180.

15. A. Ozawa et al., Phys. Rev. Lett. 84 (2000) 5493.

16. C. Nociforo et al., Phys. Lett. B 605 (2005) 79.

17. M. Stanoiu et al., Phys. Rev. C 69 (2004) 034312.

18. T. Otsuka et al., Phys. Rev. Lett. 87 (2001) 082502; A. Obertelli et al., Phys. Rev. C 71 (2005) 024304.

19. L.K. Fifield et al., Nucl. Phys. A 437 (1985) 141; P. Baumann et al., Phys. Rev. C 39 (1989) 626.

20. H. Iwasaki et al., Phys. Lett. B 620 (2005) 118; M. Belleguic et al., Phys. Rev. C 72. (2005) 054316;

21. P. Dolegeviez et al., Nucl. Instr. and Meth. 87 (2001) 08250.

22. H. Savajols and the VAMOS collaboration, Nucl. Phys. A 654 (1999) 1027c.

23. A. Obertelli et al., submitted to Phys. Rev. C.

24. B.A. Brown and B.H. Wildenthal, Annu. Rev. Nucl. Part. Sci. 38 (1988) 29.

25. N. Austern et al., Phys. Reports 154 (1987) 124.

26. A. Obertelli et al., Phys. Lett. B 633 (2006) 33.

27. Zs. Dombradi et al., Phys. Rev. Lett. 96 (2006) 182501.

28. J.R. Terry et al., Phys. Lett. B 640 (2006) 86.

29. Y. Utsuno et al., Phys. Rev.. C 70 (2004) 044307.

30. T. Otsuka et al., Phys. Rev. Lett. 95 (2005) 232502. 